\title{
A health economics study of long-acting injectable once-monthly paliperidone palmitate in schizophrenia: a one-year mirror-image study in China
}

\author{
Jie Liư ${ }^{\dagger}$, Qian Wang ${ }^{\dagger}$, Lei Su, Limin Yang, Lianyong Zou and Ludong Bai
}

\begin{abstract}
Schizophrenia is ranked among the top 25 leading causes of disability worldwide in 2013 which resulting in social and economic burden. By observing patients with schizophrenia one year before and after switching from oral antipsychotics (OAPs) to once-monthly paliperidone palmitate (PP1M), we can better understand the change of total costs in schizophrenic patients, including direct costs and indirect costs, after switching treatment patterns.

A total of 100 schizophrenic (ICD-10) patients from Shandong Mental Health Center were collected from December 2016 to June 2019. Treatment modalities, health care resource utilization and costs were compared before and after switching directly from oral antipsychotics to PP1M.

Of the 82 patients included in the main analyses, treatment with PP1M resulted in an increase in direct costs of $31.92 \%(P<0.01)$, an increase in medicine costs of approximately $142 \%(P<0.01)$, and a reduction in hospital costs of 68.15\% ( $P>0.05)$. There was no significant increase in total costs $(P=0.25)$, while $31.92 \%$ increase in direct costs $(P<$ $0.01)$, and $35.62 \%$ decrease in indirect costs $(P<0.01)$ after conversion to PP1M. Compared with before administration of PP1M, patients with $\geq 1$ inpatient stay in 1 year Pre-PP1M treatment with OAPs $(n=32)$ had a $20.16 \%$ decrease in direct costs $(P<0.01)$, a $144 \%$ increase in medicine costs $(P<0.01)$, and a significant $72.02 \%$ decrease in hospital costs $(P<0.01)$. The observed reduction in the number of hospitalizations $(t=2.56, P \leq 0.01)$ and inpatient stays $(t=1.73$, $P<0.05)$ and after transition to PP1M resulted in a reduction in hospitalization costs $(P<0.01)$.

Switching from OAPs to PP1M decreased the household workforce burden without increasing clinical healthcare costs. Direct costs were significantly reduced in patients with $\geq 1$ inpatient stay in 1 year pre-PP1M treatment with OAPs after the switch, which decreased by improving adherence to therapy and reducing the number and length of hospital stays, suggesting that those patients may benefit after switching to PP1M.
\end{abstract}

Keywords: Schizophrenia, Direct costs, Indirect costs, Health economics, Once-monthly paliperidone palmitate

*Correspondence: sanshuiju163@163.com

${ }^{\dagger}$ Jie Liu and Qian Wang both are the joint first authors of the paper.

Shandong Mental Health Center, No.49 Wenhua East Road, 250014 Jinan,

Shandong, People's Republic of China

\section{Introduction}

Schizophrenia is a remitting and relapsing psychiatric disorder, characterized by profound disruptions in thinking, language, perception, and self-perception [1]. Schizophrenia is ranked among the top 25 leading causes of disability worldwide in 2013 [2]. The average age of onset of the disease is between 15 and 35 years, usually 
in late adolescence or in the early $20 \mathrm{~s}$ in men and later in women [1]. Despite its low prevalence, its social and economic burden remains substantial, not only for patients, but also for families, and more broadly for society. Economic burden studies often incorporate both direct and indirect costs. Direct costs are costs associated with hospital inpatient treatment, prescription medications, long-term institutional care, and treatment costs associated with psychological and physical complications [3]. Indirect costs are defined as morbidity, premature mortality, and productivity losses related to informal care provided by caregivers with schizophrenia [4-7]. There are two main reasons causing the high economic burden of schizophrenia. First, non-adherence to antipsychotic use is a serious contributor to the economic burden of schizophrenia. Approximately one-third of patients with schizophrenia have poor adherence to treatment with OAPs [2]. Non-adherence to treatment can hinder the success of treatment and may lead to poor clinical outcomes and a higher risk of relapse and rehospitalization [8-11], which is also associated with increased direct cost. Second, the impairment of physical function and quality of life in schizophrenia, which often leads many nursing responsibilities for family members [12] and increases indirect burden. In fact, one study has shown that between 50 and $80 \%$ of schizophrenic patients live closely with family members [12]. Furthermore, there is a third type of costs is referred to as an intangible fee. These are associated with decreased quality of life of patients, families, and caregivers due to other factors such as pain or distress [13]. But these costs are extremely difficult to quantify and are therefore often ignored in economic research [14].

In addition, long-acting injectable antipsychotics (LAIs) have been shown to increase medication adherence [15, 16], reduce relapse and rehospitalization [16-18], and are even more cost-effective than OAPs. Although there is much strong evidence recommending the use of LAIs, it remains underutilized in clinical practice $[19,20]$ due to the so-called high costs [21]. Paliperidone palmitate is an antipsychotic in the form of a long-acting injectable formulation approved for the treatment of schizophrenia and schizoaffective disorders [22]. PP1M is intended for once-monthly intramuscular injection and it does not require any oral supplementation [23, 24], After injection, PP1M slowly dissolves owing to its extremely low water solubility, and absorbed into the systemic circulation [25]. There are currently several second-generation antipsychotic long-acting injections approved in China as options. However, PP1M was the once-monthly injectable second-generation antipsychotic approved in our hospital during our study. This study was conducted because we believe the following questions remain: which clinical treatment is more economical between OAPs and
LAIs. Based on which treatment is more cost-effective, the goal of our study is understanding the change of total costs in schizophrenic patients, including direct costs and indirect costs, after switching treatment from PP1M to OAPs. We hope to provide data support for the efficient and reasonable utilization of social health resources and for the reduction of social medical treatment costs.

\section{Methods \\ Subjects}

A total of 100 patients with schizophrenia (ICD-10) who could be followed for at least one year in Shandong Mental Health Center were collected from December 2016 to June 2019. Patients with $\geq 1$ hospitalization experience in one year before mirror point were included in the statistical analysis. The inclusion criteria were as follows: (a) between 18 and 65 years old; (b) subjects with the course of the disease $\geq 1$ year; (c) subjects were in stable condition (Positive and Negative Syndrome Scale (PANSS) total score $<70$ or Clinical Global Impression-Severity (CGI-S) score of $\leq 3$ (mild ill); (d) subjects with at least one illness exacerbation or hospitalization (PANSS score $\geq 70$ ) in the year prior to enrollment; (e) the date of the first observation of a diagnosis of schizophrenia during this period was identified as the initial date; (f) subjects received paliperidone palmitate at their own will; (g) taking antipsychotic drugs $\geq 6$ months within in one year before mirror point. The exclusion criteria were as follows: (a) pregnant or lactating women; (b) alcoholism or substance abuse; (c) patients with severe physical diseases and organic brain disease;(d) patients who could not be followed for least 1 year; (e) patients with PP1M no less than 4 times/year. We obtained data from hospital information management system. The study protocol was reviewed and approved by Ethical Committee (2017R22).

\section{Design and Variables}

This was a mirror-image study. The day on which PP1M commenced was set as the "mirror point", as show in Table 1; Fig. 1 . The study compared the number of days of health care use and costs during the 1-year period before and after PP1M implementation between December 2016 and June 2019.

Until now, direct costs were obtained through the databases. At present, objective indirect cost assessment tools are lacking. Through literature review,indirect cost is

Table 1 Disposition of models and periods

\begin{tabular}{lll}
\hline $\begin{array}{l}\mathbf{1} \text { years treatment with oral } \\
\text { antipsychotic }\end{array}$ & 1st injection & $\begin{array}{l}\mathbf{1} \text { years treatment with } \\
\text { paliperidone pamitate }\end{array}$ \\
\hline Period A & Mirror point & Period B \\
\hline
\end{tabular}




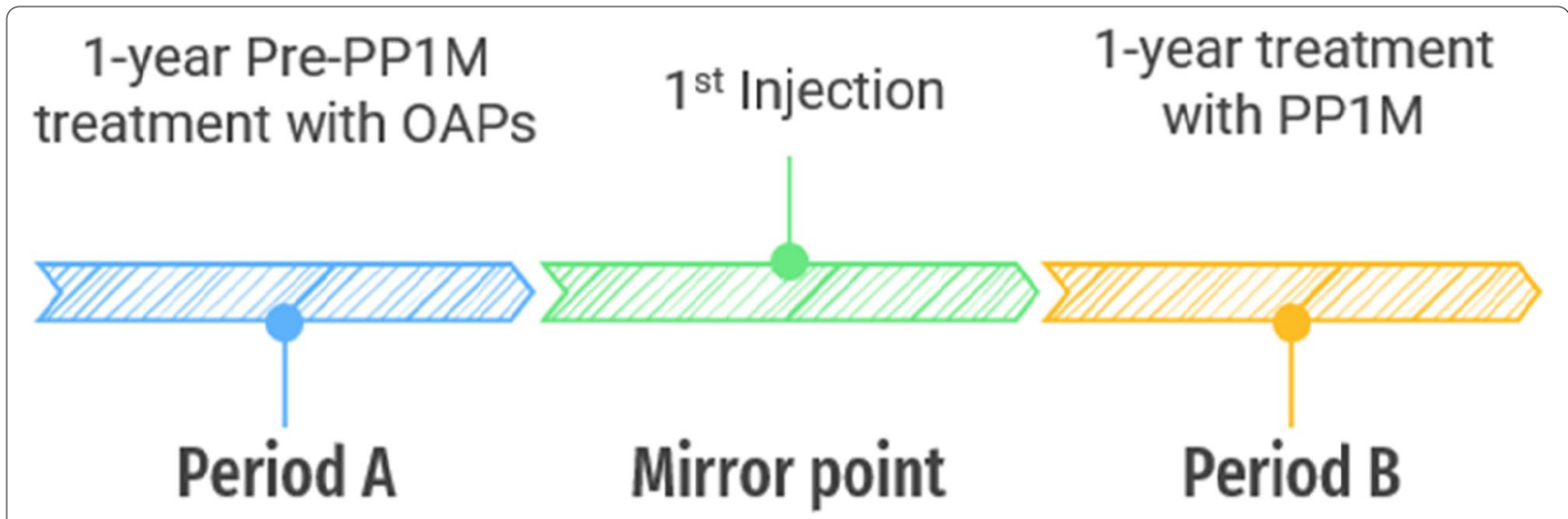

Fig. 1 Disposition of models and periods

mainly evaluated by purely descriptive method or by the author's own design of burden questionnaire,which was quantified in monetary form or in days of loss [26, 27]. In this study, we designed the economic burden scale by referring to relevant literature [23, 24]. The Economic Burden Scale assessed economic indicators where direct costs and indirect costs were quantified. Direct costs including medication and hospital costs were obtained by hospital information management system. Indirect costs included productivity costs which quantified by patient productivity loss due to psychiatric illness and caregiver care costs which quantified by caregiver loss due to patient care.

\section{Statistical methods}

Statistical analyses were performed using 23.0 SPSS software. Demographic characteristic including age, gender and mean duration of disease were described at baseline. Means, medians, and standard deviations (SDs) were used for continuous variables with T-test, while counts and percentages were used for categorical variables. Pair Wilcoxon signed-rank tests were performed to compare differences in direct (indirect)and the total costs between the pre/post-PP1M periods in this 1-year mirror-image study. Pair $\mathrm{T}$ tests were performed to compare differences in number of hospitalization and inpatient stays during the period before and after PP1M.

\section{Results}

A total of 100 patients with schizophrenia who transitioned from oral risperidone or oral paliperidone to PP1M were included in the study. 18 patients fell off, including that 7 patients lost contact, 2 patients became agitated and could not tolerate side effects, and 9 patients were unwilling to continue use PP1M for personal reasons. Patients with $\geq 1$ hospitalization experience in one year before mirror point were 32 .

\section{Demographic Before Transition to PP1M}

Of the 82 patients included in the main analyses, the average age was $32.2(\mathrm{SD}=1.2)$ years old and the mean duration of disease was 57.5 months $(\mathrm{SD}=40.6) 53.66 \%$ of the patients were female and the mean CGI-S score was $2.83(\mathrm{SD}=0.39)$.

\section{Direct Costs Before and After Transition to PP1M}

Compared with pre-PP1M, medicine costs increased significantly post-PP1M $(\mathrm{P}<0.01)$, while the hospitalization costs decreased $68.15 \%$ from $¥ 9,155$ to $¥ 2,915$ ( $\mathrm{P}>$ $0.05)$. To sum up, direct costs increased significantly from $¥ 17,457$ to $¥ 23,030(\mathrm{P}<0.01)$, as shown in Table 2 .

\section{Direct Costs for Patients with $\geq 1$ Hospitalization in 1 Year Pre-PP1M Treatment with OAP}

Compared with pre-PP1M, medicine costs in inpatient stay costs increased post-PP1M $(\mathrm{P}<0.01)$, and the hospital costs decreased significantly from $¥ 25,656$ to $¥ 7,179$ $(\mathrm{P}<0.01)$. An obvious decrease in direct costs of $\geq 1$ hospitalization in 1 year pre-PP1M treatment with OAPs were observed $(\mathrm{P}<0.01)$, as shown in Table 3.

Table 2 Wilcoxon sign-rank test was used to test the difference between pre-PP1M and Post-PP1M

\begin{tabular}{llll}
\hline & Medicine costs & $\begin{array}{l}\text { Hospitalization } \\
\text { costs }\end{array}$ & Direct costs \\
\hline pre-PP1M $(¥)$ & $8302 \pm 4156$ & $9155 \pm 18816$ & $17457 \pm 17414$ \\
Post-PP1M $(¥)$ & $20121 \pm 4580$ & $2915 \pm 8466$ & $23030 \pm 8648$ \\
Difference & $142 \%$ & $-68.15 \%$ & $31.92 \%$ \\
P & $<0.01$ & 0.074 & $<0.01$ \\
\hline
\end{tabular}


Table 3 Wilcoxon sign-rank test was used to test the difference between pre-PP1M and Post-PP1M. Comparison of Direct Cost for patients with $\geq 1$ Inpatient Stay in 1 Year Pre-PP1M Treatment with OAPs

\begin{tabular}{llll}
\hline & Medicine costs & Hospitalization costs & Direct costs \\
\hline pre-PP1M (¥) & $8992 \pm 3414$ & $25,656 \pm 18,756$ & $34,700 \pm 18,044$ \\
Post-PP1M (¥) & $21,961 \pm 5492$ & $7179 \pm 10,552$ & $27,705 \pm 8266$ \\
Difference & $144 \%$ & $-72.02 \%$ & $-20.16 \%$ \\
P & $<0.01$ & $<0.01$ & $<0.01$ \\
\hline
\end{tabular}

Table 4 Comparison of Length of Stay and Number of Hospitalizations After Conversion to PP1M

\begin{tabular}{lll}
\hline & $\begin{array}{l}\text { Number of } \\
\text { hospitalizations }\end{array}$ & Inpatient stays (days) \\
\hline pre-PP1M & $0.67 \pm 0.96$ & $52.80 \pm 60.54$ \\
Post-PP1M & $0.22 \pm 0.42$ & $16.33 \pm 29.48$ \\
$\mathrm{~T}$ & 2.56 & 1.73 \\
$\mathrm{P}$ & $\leq 0.01$ & $<0.05$ \\
\hline
\end{tabular}

Table 5 Comparison of Length of Inpatient Stay and Number of Hospitalizations After Switching to PP1M in Patients with $\geq 1$ Inpatient Stay in Period A

\begin{tabular}{lll}
\hline & $\begin{array}{l}\text { Number of } \\
\text { hospitalizations }\end{array}$ & Inpatient stays (days) \\
\hline pre-PP1M & $0.67 \pm 0.96$ & $52.80 \pm 60.54$ \\
Post-PP1M & $0.22 \pm 0.42$ & $16.33 \pm 29.48$ \\
T & 2.56 & 1.73 \\
P & $\leq 0.01$ & $<0.05$ \\
\hline
\end{tabular}

\section{Inpatient Stays and Number of Hospitalizations} after Transition to PP1M

After transition to PP1M, The number of hospitalizations $(\mathrm{t}=1.04, \mathrm{P}>0.05)$ and length of inpatient stay $(\mathrm{t}=1.73, \mathrm{P}>0.05)$ did not decrease significantly. Inpatient length of stay and number of hospitalizations (Table 4). Furthermore, as shown in Table 5. For patients $\geq 1$ hospitalization in period $\mathrm{A}$, the number of hospitalizations decreased $(\mathrm{t}=2.56, \mathrm{P}<0.05)$ and inpatient stays $(\mathrm{t}$ $=1.73, \mathrm{P}<0.05)$ also decreased from pre- to post-PP1M period, difference between the two period has statistical significance.

\section{Indirect Costs Before and After Transition to PP1M}

Compared with pre-PP1M, productivity costs $(\mathrm{P} \leq$ $0.01)$ and caregiver care costs $(P \leq 0.01)$ reduced postPP1M. Consequently, indirect costs also significantly reduced $(\mathrm{P}<0.01)$ (Table 6$)$.
Table 6 Wilcoxon sign-rank test was used to test the difference between pre-PP1M and Post-PP1M. Comparison of Indirect Costs Before and After Transition to PP1M

\begin{tabular}{llll}
\hline & $\begin{array}{l}\text { Caregiver care } \\
\text { costs }\end{array}$ & $\begin{array}{l}\text { Productivity } \\
\text { costs }\end{array}$ & Indirect costs \\
\hline pre-PP1M $¥)$ & $3830 \pm 3260$ & $11,767 \pm 8133$ & $15,967 \pm 10,128$ \\
Post-PP1M $(¥)$ & $2141 \pm 4044$ & $8137 \pm 8150$ & $10,279 \pm 11,878$ \\
Difference & $-44.10 \%$ & $-30.85 \%$ & $-35.62 \%$ \\
P & $\leq 0.01$ & $\leq 0.01$ & $<0.01$ \\
\hline
\end{tabular}

Table 7 Wilcoxon sign-rank test was used to test the difference between pre-PP1M and Post-PP1M. Comparison of Direct Costs, Indirect Costs and Total Costs Before and After Transition to PP1M

\begin{tabular}{llll}
\hline & Direct costs & Indirect costs & Total costs \\
\hline pre-PP1M (¥) & $17,457 \pm 17,414$ & $15,967 \pm 10,128$ & $33,095 \pm 21,984$ \\
Post-PP1M (¥) & $23,030 \pm 8648$ & $10,279 \pm 11,878$ & $33,309 \pm 15,254$ \\
Difference & 31.92 & $-35.62 \%$ & $0.64 \%$ \\
P & $<0.01$ & $<0.01$ & 0.25 \\
\hline
\end{tabular}

Table 8 Wilcoxon sign-rank test was used to test thedifference between pre-PP1M and Post-PP1M For Patients with $\geq 1$ Inpatient Stay inPeriod A , Comparison of Direct Cost, Indirect Costs and Total Costs Before andAfter Transition to PP1M

\begin{tabular}{llll}
\hline & Direct costs & Indirect costs & Total costs \\
\hline pre-PP1M (¥) & $34,700 \pm 18,044$ & $21,718 \pm 11,246$ & $56,418 \pm 28,020$ \\
Post-PP1M (¥) & $27,705 \pm 8266$ & $15,023 \pm 10,875$ & $42,728 \pm 15,307$ \\
Difference & -20.16 & $-30.83 \%$ & $-24.27 \%$ \\
P & $<0.01$ & $<0.01$ & 0.478 \\
\hline
\end{tabular}

\section{Total Costs Before and After Transition to PP1M}

The direct costs of the patients increased $(P<0.01)$, while the indirect costs decreased significantly (P $<0.05$ ) after the switch to PP1M, by the comparison of the pre-PP1M period. Overall, the total costs to patients was no significant increased $(\mathrm{P}>0.05)$, as shown in Table 7. Furthermore, as shown in Table 8. For patients $\geq 1$ hospitalization in 1 year Pre-PP1M treatment with OAPs, an obvious decrease in indirect costs $(\mathrm{P}<0.01)$ and direct costs $(\mathrm{P}<0.01)$ during the transition to PP1M were observed, the total costs decreased $27 \%$ after switching to PP1M, but there was not statistically significant $(\mathrm{P}>0.05)$.

\section{Discussion}

Nowadays, oral antipsychotics remain the first-line pharmacologic treatment option for patients with schizophrenia. However, a high discontinuation rate is a known problem with oral antipsychotic treatment [28]. LAIs are 
generally indicated in patients who are considered nonadherent to oral therapy. In this mirror-image study, after switching from OAPs to PP1M, the number of hospitalizations $(\mathrm{t}=1.04, \mathrm{P}>0.05)$ and length of inpatient stay $(\mathrm{t}$ $=1.73, \mathrm{P}>0.05)$ did not decrease significantly. The other study reported some different finding [29]. In this Germany mirror-image design [29], 119 patients with schizophrenia and schizoaffective disorder who switched to risperidone long acting injection (RLAI), after 12 and 18 months of RLAI treatment, the mean reduction of inpatient care was 27.4 and 38.4 days per patient, respectively. The different results may be related to the different subjects selected in the two studies. The patients selected in our study were in stable condition and took OAPs for more than six months before the mirror point. While, for patients $\geq 1$ hospitalization in 1 year, using OAPs for Pre-PP1M treatment, there were not only fewer number of hospitalizations but also fewer days spent in an inpatient setting. The results of this study are consistent with those of others [20, 21]. More importantly, in addition to improvements in adherence, changes in pharmacoeconomics were also found after switching to PP1M. For the treatment of schizophrenia, most of the treatment costs do come from hospitalization [30, 31].We found that medicine costs increased, while hospital costs had no significant reduction after switch from PP1M to OAPs, resulting in an increase in direct costs $(¥ 17,457$ versus $¥ 23,030, \mathrm{P}<0.01)$. However, patients who have had $\geq 1$ hospitalization experience in the year before the mirror point will have different consequences. Surprisingly, the decline in hospital costs could completely offset the increase in medicine costs, resulting a decrease in direct costs ( $¥ 34,700$ versus $¥ 27,705, \mathrm{P}<0.01$ ). We also found the same results with other research [32]. In a mirror study conducted in South Korea [32], 1272 Korean schizophrenics who switched from OAPs to PP1M were followed for 1 year: the direct costs of outpatients increased by $\$ 1497$; for inpatients, direct costs was reduced by approximately $\$ 1,220$. We found that the use of PP1M can partially reduce the economic burden of the disease for patients with $\geq 1$ hospitalization experience before mirror point. Because these patients had less number of hospitalizations and length of stay. In a real-world observational study [33], the use of LAIs therapy was associated with fewer hospitalizations and fewer hospital days, which resulted in a significant reduction in monthly hospital costs ( $\$ 4,007$ for LAIs and $\$ 8,769$ for OAPs cohort). Patients with $\geq 1$ inpatient stay pre-PP1M transition may particularly benefit from switching from OAPs to PP1M, since it improves adherence and decreases the length of stay, thereby significantly reducing direct costs.

At the same time, schizophrenia patients affected by the mental symptom caused high productivity loss, and resulted in high productivity losses to patients and their families. In addition, the burden of schizophrenia, in addition to direct medicine costs also had a profound impact on caregivers and patient families [34]. Currently, few studies have observed changes in indirect costs of patients with schizophrenia from OAP to PP1M. This study found that indirect costs (productivity loss and informal care costs provided by caregivers) were effectively reduced when patients were switched from OAPs to PP1M ( $\leq 0.01)$. In the meanwhile, the quality of life of caregivers may also be improved. In summary, this study found that after the transition from OAPs to PP1M, direct costs have increased while indirect costs have decreased, resulting in a further reduction in no difference in total costs (after from OAPs transition to PP1M.

In contrast to prior studies that included a comparison of OAPs to PP1M using a cohort design, this study used a mirror-image design to compare patients before and after the initiation of PP1M. The mirror-image design is a study that compare the results before and after the medication changes (from OAPs to PP1M). The focus here is to compare the economic results of dressing changes for patients with schizophrenia before and after. There are not many studies in this direction, and previous literature review studies have focused on summarizing direct costs associated with schizophrenia. There has been a lack of emphasis on indirect costs estimation. Furthermore, China has no similar results at present. Therefore, this study focuses on the economic burden of direct and indirect costs when Chinese patients with schizophrenia switch from OAP to PP1M.

However, the finding of this study should be interpreted in the context of some limitations. First, part of our database (indirect costs) was based on patient-provided data, and its accuracy cannot be guaranteed. Second, the sample size was relatively small, and the data may not be representative of the entire Chinese population. Therefore, in the follow-up study, we will further cooperate with other regions in China to carry out follow-up research and evaluation.

\section{Conclusions}

Comparing patients one year before and after switching from OAPs to PP1M, the direct costs increased and the indirect costs decreased, leading to a smaller difference in total costs after switching to PP1M. This suggests that patients switching from OAPs to PP1M can improve patient productivity, reduce caregiver burden and can not increase the costs of health care in the clinic. For patients with $\geq 1$ inpatient stay one year prior to PP1M, the hospital costs decreased which were associated with the reduction of number of hospitalization and inpatient stays, and the medicine costs increased which was fully offset by 
lower hospital costs after switching from OAPs to PP1M. The reduction of direct costs suggests that patients with hospitalization experience obtain greater economic benefits by changing from OAPs to PP1M.

\author{
Abbreviations \\ OAPs: oral antipsychiotics; LAls: long-acting injectable antipsychotics; PP1M: \\ once-monthly paliperidone palmitate; OAPs: oral antipsychotics; ICD-10: \\ international classification of diseases, tenth revison; CGI-S: clinical global \\ impressions scale; PANSS: positive and negative syndrome scale; SD: standard \\ deviation; RLAl: risperidone long acting injection.
}

\section{Acknowledgements}

We appreciate all participants of the study and our families for their supports.

\section{Authors' contributions}

Bai designed the study. Liu and Wang performed the data analyses and wrote the manuscript. Liu and Wang contribute equally. Su contributed significantly to analysis and manuscript preparation. Yang and Zou helped perform the analysis with constructive discussions. The author(s) read and approved the final manuscript.

\section{Funding}

The study supported by grants from the Shandong Medical and Health Science and Technology Development project (Grant No.2017 WS792) and Shandong science and technology project of traditional Chinese medicine (Grant No.2020M053).

\section{Availability of data and materials}

All data generated or analyzed during this study are included in this published article. Data are available on request from the corresponding author.

\section{Declarations}

\section{Ethics approval and consent to participate}

The study was conducted in accordance with the ethical principles of the Declaration of Helsinki, consistent with Good Clinical Practices and was approved by the Human Ethics Committee of Shandong Mental Health Center (2017R22). Written informed consent was obtained from individual or guardian participants after them understood the purpose, risks and benefits of the study.

\section{Consent for publication}

Not applicable.

\section{Competing interests}

Not applicable.

Received: 14 June 2021 Accepted: 18 January 2022

Published online: 08 February 2022

\section{References}

1. Goldner EM, Hsu L, Waraich P, Somers JM. Prevalence and incidence studies of schizophrenic disorders: a systematic review of the literature. Can J Psychiatry. 2002;47:833-43. https://doi.org/10.1177/0706743702 04700904.

2. Correll CU, et al. The Use of Long-Acting Injectable Antipsychotics in Schizophrenia: Evaluating the Evidence. J Clin Psychiatry. 2016;77:1-24. https://doi.org/10.4088/JCP.15032su1.

3. Ng CS, Lee JY, Toh MP, Ko Y. Cost-of-illness studies of diabetes mellitus: a systematic review. Diabetes Res Clin Pract. 2014;105(2):151-163

4. Leardini G, Salaffi F, Caporali R, Canesi B, Rovati L, Montanelli R. Direct and indirect costs of osteoarthritis of the knee. Clin Exp Rheumatol. 2004;22(6):699-706.
5. Castro DM, Dillon C, Machnicki G, Allegri RF. The economic cost of Alzheimer's disease: family or public health burden? Dement Neuropsychol. 2010;4(4):262-267.

6. Filipovic I, Walker D, Forster F, Curry AS. Quantifying the economic burden of productivity loss in rheumatoid arthritis. Rheumatology (Oxford) 2011;50:1083-1090.

7. Dadoun S, Guillemin F, Lucier S, et al. Work productivity loss in early arthritis during the first 3 years of disease: a study from a French national multicenter cohort. Arthritis Care Res. 2014;66(9):1310-1318.

8. Ascher-Svanum $\mathrm{H}$, et al. Medication adherence and long-term functional outcomes in the treatment of schizophrenia in usual care. J Clin Psychiatry. 2006;67:453-60. https://doi.org/10.4088/jcp.v67n0317.

9. Haddad PM, Brain C, Scott J. Non-adherence with antipsychotic medication in schizophrenia: challenges and management strategies. Patient Relat Outcome Meas. 2014;5:43-62. https://doi.org/10.2147/ PROM.S42735.

10. MacEwan JP, et al. Patterns of Adherence to Oral Atypical Antipsychotics Among Patients Diagnosed with Schizophrenia. J Manag Care Spec Pharm. 2016;22:1349-61. https://doi.org/10.18553/jmcp.2016.22.11. 1349.

11. Novick D, et al. Predictors and clinical consequences of non-adherence with antipsychotic medication in the outpatient treatment of schizophrenia. Psychiatry Res. 2010;176:109-13. https://doi.org/10.1016/j. psychres.2009.05.004.

12. Saraceno $B$, et al. Barriers to improvement of mental health services in low-income and middle-income countries. Lancet. 2007;370:1164-74. https://doi.org/10.1016/S0140-6736(07)61263-X.

13. Kleine-Budde $K$, et al. Cost of illness for bipolar disorder: a systematic review of the economic burden. Bipolar Disord. 2014;16:337-53. https://doi.org/10.1111/bdi.12165.

14. Cooper NJ. Economic burden of rheumatoid arthritis: a systematic review. Rheumatology (Oxford). 2000;39:28-33. https://doi.org/10. 1093/rheumatology/39.1.28.

15. Greene $\mathrm{M}$, et al. Medication adherence and discontinuation of longacting injectable versus oral antipsychotics in patients with schizophrenia or bipolar disorder. J Med Econ. 2018;21:127-34. https://doi. org/10.1080/13696998.2017.1379412.

16. Marcus SC, Zummo J, Pettit AR, Stoddard J, Doshi JA. Antipsychotic Adherenceand Rehospitalization in Schizophrenia Patients Receiving Oral Versus Long-Acting Injectable Antipsychotics Following Hospital Discharge. J Manag Care Spec Pharm. 2015;21:754-68. https://doi.org/ 10.18553/jmcp.2015.21.9.754.

17. Tiihonen J, et al. Real-World Effectiveness of Antipsychotic Treatments in a Nationwide Cohort of 29823 Patients With Schizophrenia. JAMA Psychiatry. 2017;74:686-93. https://doi.org/10.1001/jamapsychiatry. 2017.1322.

18. Taipale H, Mehtala J, Tanskanen A, Tiihonen J. Comparative Effectiveness of Antipsychotic Drugs for Rehospitalization in Schizophrenia-A Nationwide Study With 20-Year Follow-up. Schizophr Bull. 2018;44:1381-7. https://doi.org/10.1093/schbul/sbx176.

19. West JC, et al. Use of depot antipsychotic medications for medication Non-adherence in schizophrenia. Schizophr Bull. 2008;34:995-1001. https://doi.org/10.1093/schbul/sbm137.

20. Lafeuille MH, Grittner AM, Fortier J, et al. Comparison of rehospitalization rates and associated costs among patents with schizophrenia receiving paliperidone palmitate or oral antipsychotics. Am J Health Syst Pharm.2015:72:378-389.

21. Correll CU, Citrome L, Haddad PM, et al. The use of long-acting injectable antipsychotics in schizophrenia: evaluating the evidence. J Clin Psychiatry.2016:77:1-24.

22. Giday E, Nasrallah HA. Clinical pharmacology of paliperidone palmitate long-acting formulation for the treatment of schizophrenia. Recent Clin Trials. 2012;7(1):2-9.

23. Owen RT. Paliperidone palmitate injection: its efficacy, safety and tolerability in schizophrenia. Drugs Today. 2010;46(7):463-471.

24. Citrome L. Paliperidone palmitate - review of the efficacy, safety and cost of a new second-generation depot antipsychotic medication. Int J Clin Pract. 2010;64(2):216-239.

25. M. C. Mauri, et al,. Paliperidone for the treatment of schizophrenia and schizoaffective disorders a drug safety evaluation. Expert Opinion on Drug Safety, 2017;16(3):365-379. 
26. Zivin K, Bohnert AS, Mezuk B, et al. Employment status of patients in the VA health system: implication for mental health services. Psychiatr Serv.2011;62(1):35-38.

27. Goeree R, O'Brien BJ, Blackhouse G, et al. The valuation of productivity costs due to premature mortality: a comparison of the human-capital and friction-cost methods for schizophrenia. Can J Psychiatry.1999:44(5):455-463

28. Lieberman JA, et al. Effectiveness of antipsychotic drugs in patients with chronic schizophrenia. N Engl J Med. 2005;353:1209-23. https://doi.org/ 10.1056/NEJMoa051688.

29. Birgit, Spill, et al. Long-term observation of patients successfully switched to risperidone long-acting injectable: A retrospective, naturalistic 18-month mirror-image study of hospitalization rates and therapy costs. Int J Psychiatry Clin Pract. 2010;14:53-62.

30. Davies, L. M. \& Drummond, M. F. Economics and schizophrenia: the real cost. Br J Psychiatry Suppl. 1994; 18-21.

31. Chue PS, Heeg B, Buskens E, van Hout BA. Modelling the impact of compliance on the costs and effects of long-acting risperidone in Canada. Pharmacoeconomics. 2005;23(Suppl 1):62-74. https://doi.org/10.2165/ 00019053-200523001-00006.

32. Lee Dasom, et al. Effects of paliperidone palmitate on healthcare utilization and costs for patients with schizophrenia: A claim-based mirror-image study in South Korea. Clin Psychopharmacol Neurosci. 2020;18(2):303.

33. Ankit Shah, et al. Treatment Patterns, Healthcare Resource Utilization and Costs Among Schizophrenia Patients Treated with Long-Acting Injectable Versus Oral Antipsychotics. Adv Ther 2018.

34. Tajima-Pozo K, de Castro Oller MJ, Lewczuk A, Montanes-Rada F. Understanding the direct and indirect costs of patients with schizophrenia. F1000Res. 2015;4:182. https://doi.org/10.12688/f1000research.6699.2.

\section{Publisher's Note}

Springer Nature remains neutral with regard to jurisdictional claims in pub-

lished maps and institutional affiliations.

- fast, convenient online submission

- thorough peer review by experienced researchers in your field

- rapid publication on acceptance

- support for research data, including large and complex data types

- gold Open Access which fosters wider collaboration and increased citations

- maximum visibility for your research: over $100 \mathrm{M}$ website views per year

At BMC, research is always in progress.

Learn more biomedcentral.com/submissions 